\title{
Coronavirus COVID-19 y dolor crónico: incertidumbres
}

\author{
COVID-19 coronavirus and chronic pain: uncertainties
}

La pandemia producida por el coronavirus COVID-19 ha conducido a los ciudadanos al aislamiento, o "distanciamiento social", con el fin de impedir su propagación. Varios de ellos probablemente son pacientes con dolor crónico y de seguro muchos tienen una edad avanzada, un factor de riesgo añadido, como estamos viendo trágicamente cada día. Conocido es también que la presencia de dolor interfiere con las actividades de la vida diaria en el $14 \%$ de la población, incrementándose hasta un $20 \%$ en los mayores de 65 años, lo que no deja de ser importante en situaciones de aislamiento.

A este respecto, la última encuesta de salud de la población española realizada por el Ministerio de Sanidad [1] arroja cifras que confirman la importancia del dolor. En efecto, se observa una prevalencia de artrosis en el $18 \%$ de la población, cervicalgia en el $16 \%$ y en dolor lumbar crónico un $19 \%$; adicionalmente, los procesos oncológicos afectan al $1,8 \%$ de la población. Esta fuente oficial nos ofrece también otros datos de interés con relación al dolor: así, por ejemplo, se informa que el dolor está presente en las últimas cuatro semanas en el $45 \%$ de la población, siendo de intensidad extrema o severa en el $9 \%$, llegando al $15 \%$ en los mayores de 75 años, franja de edad más castigada en la pandemia por coronavirus en España.

De estos datos se puede inferir que las necesidades de fármacos analgésicos son elevadas. En efecto, los informes de la Agencia española de Medicamentos indican que los analgésicos simples y los antinflamatorios representan las principales categorías de consumo de fármacos [2], y se estima que un $13 \%$ de la población consume opioides [3].

Algunos de estos fármacos han producido en estos días de pandemia una ola de informaciones sobre su uso, creando alertas entre los pacientes y los profesionales de la salud. Afortunadamente, estas cuestiones se han ido esclareciendo mediante informes y comunicados emitidos por diferentes agencias, tanto nacionales (AEMPS) como internacionales [OMS] $[4,5]$. Desde la SED también hemos ido informando puntualmente [www.sedolor. es], difundiendo asimismo cuantas medidas de protección personal y comunitaria contra la infección se han ido publicando, siempre desde fuentes de garantía.

Es de destacar también que, en redes sociales, no han sido pocos los pacientes con dolor crónico que han emitido mensajes informando del deterioro de su salud, tanto física como mental, como consecuencia del aislamiento o distanciamiento social.

En definitiva, vemos que, durante la pandemia, y con seguridad también cuando volvamos a la normalidad, los pacientes con dolor crónico presentan unas características que merecen y merecerán la atención de la salud pública española en todas sus vertientes.

Diversos estudios, tanto clínicos como experimentales y sociales, han centrado su interés desde hace tiempo en estas situaciones a las que están sometidos los pacientes con dolor crónico, señalando que la soledad, el distanciamiento social y la incertidumbre en la prestación de cuidados de salud, no son buenos para el pronóstico y curso de un proceso doloroso $[6,7]$.

El dolor como un factor estresante y sus dimensiones sensorial y emocional se relacionan muy estrechamente, y hay varios factores tanto psicológicos como sociales y neurobiológicos que condicionan este círculo tan difícil a veces de romper, a no ser que se haga mediante una aproximación terapéutica biopsicosocial [8].

Cabe preguntarse cuáles serían los factores que hacen que una situación emocional anómala, como es el aislamiento o el distanciamiento social de larga duración, agrave un cuadro doloroso. También nos preguntamos si el estrés que ocasiona esta situación, la ansiedad e incluso la depresión que se genera contribuyen negativamente a cerrar este círculo vicioso, lo que sin duda ocurre [9]. De hecho, los pacientes con dolor crónico que 
tengan que estar, como cualquier ciudadano, en aislamiento/distanciamiento social debido a la pandemia por el COVID-19 pueden empeorar su cuadro físico y emocional.

En otra dimensión, se formulan otras cuestiones tales como si los medicamentos analgésicos o coadyuvantes que estén tomando los pacientes COVID-19 positivos pueden afectar su inmunidad o cualquier otro proceso fisiológico y, por ende, ser perjudicial en estas condiciones de aislamiento y en el propio proceso patológico que sufre. También es necesario conocer las posibles interacciones de estos fármacos analgésicos y coadyuvantes con la terapéutica farmacológica específica aplicada a los pacientes con COVID-19 positivo y afectados por la enfermedad.

En este último aspecto, mientras estos dilemas clínicos y terapéuticos se dirimen, se puede acudir a información específica sobre estas interacciones medicamentosas en direcciones de garantía, tales como https://www.hep-druginteractions.org/checker.

En definitiva, por ahora es complicado responder a estas cuestiones con suficiente respaldo científico y clínico, lo que abre, dentro de la problemática situación que estamos viviendo, un amplio abanico de posibilidades que deberán obligatoriamente ser exploradas rigurosamente y estar preparados para un (esperemos que no) rebrote de esta u otra pandemia vírica similar.

Desde la RESED, desearíamos animar a nuestros lectores a aportar manuscritos originales, revisiones, casos clínicos, guías o cualquier otro formato, que puedan contribuir a mejorar el conocimiento de la interrelación del dolor con la pandemia COVID-19, de tal manera que, si no es posible ahora, sí tengamos para el futuro preparados argumentos clínicos y científicos rigurosos suficientes para contrarrestar los problemas que nos puedan surgir, y esperemos que no nos hagan falta.

Un cordial saludo y nuestro mayor reconocimiento a todos los profesionales de la salud que luchan cada día contra esta pandemia y nuestro pésame a las familias y allegados de los que han perdido su vida por salvar la de otros.

\section{J. A. Micó Segura Departamento de Neurociencias. Facultad de Medicina. Universidad de Cádiz, España. Presidente de la Sociedad Española del Dolor}

Correspondencia: Juan Antonio Micó juanantonio.mico@uca.es

\section{BIBLIOGRAFÍA}

1. Ministerio de Sanidad. Encuestra Nacional de Salud de Espala 2017. Disponible en: https://www.mscbs. gob.es/estadEstudios/estadisticas/encuestaNacional/encuesta2017.htm

2. Ministerio de Sanidad, Servicios Sociales e Igualdad. Utilización de medicamentos antiinflamatorios no esteroideos en España durante el periodo 2013-2016. Publicado el 22 de septiembre de 2017. Disponible en: https://www.aemps.gob.es/medicamentosUsoHumano/observatorio/docs/antiinflamatoriosAINEs-periodo-2013-2016.pdf?x33378.

3. Álvarez Mazariegos JA, Calvete Waldomar S, Fernández-Marcote Sánchez-Mayoral RM, Guardia Serecigni J, Henche Ruiz Al, Lligoña Garreta A, et al. Guía de Consenso para el buen uso de analgésicos opioides. Gestión de riesgos y beneficios. Valencia: Socidrogralcohol; 2017. Disponible en: http://www. pnsd.mscbs.gob.es/profesionales/publicaciones/catalogo/bibliotecaDigital/publicaciones/pdf/2017_ GUIA_Buen_uso_opioides_Socidrigalcohol.pdf

4. Ministerio de Sanidad. La AEMPS informa de que ningún dato indica que el ibuprofeno agrave las infecciones por COVID-19. Publicado 15 de marzo de 2020. Disponible en: https://www.aemps.gob.es/ informa/notasinformativas/medicamentosusohumano-3/2020-medicamentosusohumano-3/la-aempsinforma-que-ningun-dato-indica-que-el-ibuprofeno-agrave-las-infecciones-por-covid-19/

5. World Health Organization. Disponible en: https://twitter.com/WHO/status/1240409217997189128? ref_src=twsrc\%5Etfw\%7Ctwcamp\%5Etweetembed\%7Ctwterm\%5E1240409217997189128\&ref_ url=https\%3A\%2F\%2Fwww. sciencealert.com\%2Fwho-recommends-to-avoid-taking-ibuprofen-for-covid19-symptoms

6. Dueñas M, Ojeda B, Salazar A, Mico JA, Failde I. A review of chronic pain impact on patients, their social environment and the health care system. J Pain Res. 2016;9:457-67. 2016. DOI: 10.2147/ JPR.S105892.

7. Solé E, Racine M, Tomé-Pires C, Galán S, Jensen MP, Miró J. Social Factors, Disability and Depressive Symptoms in Adults with Chronic Pain. Clin J Pain. 2020. DOl: 10.1097/AJP.0000000000000815. [Epub ahead of print]

8. Micó JA, Berrocoso E. Influence of chronic stress on the somatic and emotional dimensions of chronic pain. Pain 2016. Refresher Courses. 16th World Congress of Pain. Claudia Sommer, Mark S Wallace, Steven P. Cohen and Michaela Kress, editors. Washington, D.C.: IASP Press, p. 399-404.

9. Blackburn-Munro G, Blackburn-Munro RE. Chronic pain, chronic stress and depression: coincidence or consequence?. J Neuroendocrinol. 2001;13(12):1009-23. DOI: 10.1046/j.0007-1331.2001.00727.x. 